\section{Semi-Synthesis of Kaurenoic Acid Derivatives and Their In Vitro Cytotoxic Activities}

\author{
Lei Zhang ${ }^{1}$, Fei Wang ${ }^{1}$, Xin-hua Ma ${ }^{1}$, Fang Zhou ${ }^{1}$, Sheng-mei \\ Wen ${ }^{1}$, Tian-hua Zhong ${ }^{2}$, Quan-yu Liu ${ }^{1}$, Shi-wu Chen ${ }^{3}$, \\ Yong-hong Zhang ${ }^{1}$ \\ ${ }^{1}$ Key Laboratory of Natural Drug Pharmacology in Fujian Province, \\ School of Pharmacy, Fujian Medical University, Fuzhou, P. R. China \\ 2 Key Laboratory of Marine Biogenetic Resources, Third Institute of \\ Oceanography, State Oceanic Administration, Xiamen, P. R. China \\ 3 School of Pharmacy, Lanzhou University, Lanzhou, P. R. China
}

\section{Abstract \\ $\nabla$}

The cytotoxic activities of the diterpene kaurenoic acid (1) and its 15 semi-synthesis derivatives were assessed on human cell cultures. The human tumor cells used comprised colon (SW620 and SW480), pancreatic (PANC-1 and BxPC-3), stomach (SGC-7901), esophageal (Eca-109), and leukemia (K562 and HL-60). Kaurenoic acid was inactive against the tumor cell lines; however, its derivatives which contain $\alpha, \beta$-unsaturated ketone rendered compounds with cytotoxic activity. Compounds 5-14, 17-19, and 24 with a substitution at the $C-4$ position showed significant inhibitory activity against the tested cell lines, while compound $\mathbf{3}$, without a substitution at the C-4 position, was slightly less active in these cell lines. The SW620 colon cancer cell was highly susceptible to all of the tested derivatives.

\section{Key words}

Wedelia prostrata - Asteraceae - kaurenoic acid derivatives . semi-synthesis · cytotoxic activity $\cdot$ cancer cell lines

$\begin{array}{ll}\text { Abbreviations } \\ \text { BrR: } & \text { alkyl bromide } \\ \text { Br-R-Br: } & \text { dibromoalkanes } \\ \text { DCC: } & \text { dicyclohexylcarbodiimide } \\ \text { DMF: } & \text { dimethylformamide } \\ \text { DMSO: } & \text { dimethylsulfoxide } \\ \text { EI-MS: } & \text { electron impact mass spectrometry } \\ \text { EtBr: } & \text { bromine ethane } \\ \text { EtOH: } & \text { ethanol } \\ \text { HOBt: } & \text { hydroxybenzotriazole } \\ \text { HR-MS: } & \text { high resolution mass spectrometer } \\ \text { IR: } & \text { infrared spectrum } \\ \text { KI: } & \text { potassium iodide } \\ \text { MTT: } & \text { 3-(4,5-dimethyl-2-thiazolyl)-2,5-diphenyl-2-H-tetra- } \\ & \text { zolium bromide } \\ \text { OD: } & \text { optical density } \\ \text { PDC: } & \text { pyridinium dichromate } \\ \text { t-BuOOH: } & \text { peroxide tert-butyl alcohol } \\ \text { THF: } & \text { tetrahydrofuran } \\ \text { TMS: } & \text { tetramethylsilane } \\ \text { TLC: } & \text { thin layer chromatography } \\ & \end{array}$

Supporting information available online at http://www.thieme-connect.de/products
Kaurenoic acid (1) [1,2] is one of the active constituents in Wedelia prostrata (Hook. et Arn.) Hemsl. (Asteraceae) [3], a traditional Chinese herbal medicine [4]. It is an ent-kaurane diterpenoid [5], which is claimed to have important biological activities, mainly antimicrobial [6], antibacterial [7], cytotoxic [8,9], anti-inflammatory [10], and anticonvulsant properties [11]. In the present paper, we describe the semi-synthesis of kaurenoic acid derivatives and their preliminary cytotoxic activities. A conversion of the 15-hydroxy group of kaurenoic acid to a ketone is made in order to incorporate an $\alpha, \beta$-unsaturated ketone into the ent-kaurane skeleton. It is well know that a main structural determinant for cytotoxicity is present in an $\alpha, \beta$-unsaturated ketone system $[12,13]$, which likely serves as an alkylating center and can be part of an ester, ketone, or lactone moiety. We also report several transformations on the carboxylic acid group at the C-4 position. Compound $\mathbf{2}$ was obtained from 1 after treatment with $\mathrm{SeO}_{2} / \mathrm{t}-$ $\mathrm{BuOOH}$ in THF. Oxidation of the 15-hydroxy group of compound 2 using PDC produced $\alpha, \beta$-unsaturated ketone $\mathbf{3}$ (Fig. 1 S, Supporting Information). Compound $\mathbf{2}$ was amidated with $\mathrm{RNH}_{2} /$ DCC/HOBt in THF/DMF to yield $\mathbf{4 a - 4 d}$ and oxidized with PDC to yield the corresponding amide derivatives 5-8. Amide derivatives 9-10 were synthesized from $\mathbf{2}$ under treatment with pyridine/DCC and then oxidized to ketone. Ester derivatives of compounds 11-14 were obtained directly from 3 with $\mathrm{BrR} / \mathrm{K}_{2} \mathrm{CO}_{3} / \mathrm{KI}$ in DMF. Treatment of 2 with $\mathrm{K}_{2} \mathrm{CO}_{3}$ and $\mathrm{Br}-\mathrm{R}-\mathrm{Br}$ in DMF formed $15 a-15 c$, which subsequently converted into compounds 16a$16 \mathrm{c}$ under treatment with piperidine and $\mathrm{K}_{2} \mathrm{CO}_{3}$ in THF. The oxidation of 16a-16c with PDC yielded 17-19. Compound 1 was esterfied with EtBr to give 20, which was reduced with $\mathrm{LiAlH}_{4}$ to form the alcohol 21. The reaction of $\mathbf{2 1}$ with $\mathrm{Ac}_{2} \mathrm{O}$ formed the corresponding ethyl ester 22. The reaction of $\mathbf{2 2}$ with $\mathrm{SeO}_{2}$ and t$\mathrm{BuOOH}$ obtained compound $\mathbf{2 3}$ and the oxidation of $\mathbf{2 3}$ yielded 24 ( $\odot$ Fig. 1). Compound 3 has been reported previously [14], while the 14 derivatives $(\mathbf{5 - 1 4}, \mathbf{1 7 - 1 9}, \mathbf{2 4})$ were reported here for the first time. The structures of the derivatives were confirmed by ${ }^{1} \mathrm{H}-\mathrm{NMR},{ }^{13} \mathrm{C}-\mathrm{NMR}$, IR, HR-MS, EI-MS, and ESI-MS data (see Supporting Information).

The cytotoxic activities of compound 1 and its 15 semi-synthesis derivatives were assessed on eight human cell lines ( $\boldsymbol{O}$ Table $\mathbf{1}$ ). The results of the cytotoxicity assays indicated that compound $\mathbf{1}$, without the $\alpha, \beta$-unsaturated ketone, was inactive, while the 15 derivatives, which do contain this moiety, were active against all or some of the cell lines. Thus, as proposed in the literature [12, $13]$, the $\alpha, \beta$-unsaturated ketone is the active center, possibly acting as an alkylation site. Compound $\mathbf{3}$ without a substitution at the C-4 position had moderate activities to SGC-7901 and K562 with $\mathrm{IC}_{50}$ values ranging from 7.37 to $7.53 \mu \mathrm{M}$, while compounds 5-14, 17-19, and 24 with a substitution at the C-4 position showed stronger inhibitory activity than compound 3 to those tumor cell lines with $\mathrm{IC}_{50}$ values ranging from 0.25 to $2.47 \mu \mathrm{M}$. This indicated that the substitution of the acid moiety at the C-4 position led to significant changes in the cytotoxic activity. Compounds 5-7 with amide groups at C-4 displayed more potent cytotoxicity than compounds 11-14 with ester groups at C-4 against the K562 cell line but were less potent to the SGC-7901 cell line. Meanwhile, compounds 11-14 with only ester groups at C-4 showed higher cytotoxic activity than compounds 17-19 with piperidine groups conjunct to them at C-4 to SGC-7901. Therefore, different kinds of substituted groups would cause different effects to different cell lines. The cytotoxicity of compounds 11-13 with ethyl, propyl, and butyl ester groups at C-4 implied that the elongated aliphatic chain length did not influ- 
<smiles>C=C1C[C@]23CC[C@@H]4[C@@](C)(C(=O)O)CCC[C@]4(C)[C@H]2CC[C@H]1C3</smiles>

kaurenoic acid (1)

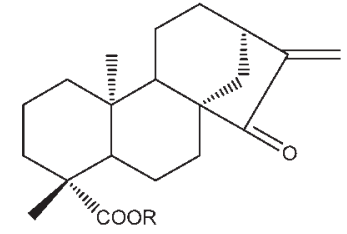

$\mathrm{R}$

$3 \mathrm{H}$

$11 \mathrm{CH}_{2} \mathrm{CH}_{3}$

$12 \quad \mathrm{CH}_{2} \mathrm{CH}_{2} \mathrm{CH}_{3}$

$13 \mathrm{CH}_{2} \mathrm{CH}_{2} \mathrm{CH}_{2} \mathrm{CH}_{3}$

$14 \mathrm{CH}_{2} \mathrm{C}_{6} \mathrm{H}_{5}$

$17 \quad\left(\mathrm{CH}_{2}\right)_{2}-\mathrm{N}$

$18 \quad\left(\mathrm{CH}_{2}\right)_{3}-\mathrm{N}$

$19 \quad\left(\mathrm{CH}_{2}\right)_{4}-\mathrm{N}$<smiles>C=C1C(O)[C@]23CCC4[C@@](C)(C(=O)O)CCC[C@]4(C)C2CC[C@H]1C3</smiles>

2<smiles>C=C1C(=O)[C@]23CCC4[C@@](C)(C(N)=O)CCC[C@]4(C)C2CC[C@H]1C3</smiles>

$\mathrm{R}$

$5 \mathrm{CH}_{2} \mathrm{CH}_{2} \mathrm{CH}_{3}$

$6 \mathrm{CH}\left(\mathrm{CH}_{3}\right)_{2}$

$7 \quad \mathrm{CH}_{2} \mathrm{CH}_{2} \mathrm{CH}_{2} \mathrm{CH}_{3}$

$8 \quad \mathrm{CH}_{2} \mathrm{C}_{6} \mathrm{H}_{5}$

9 phenylalanine methyl ester

10 valine methyl ester

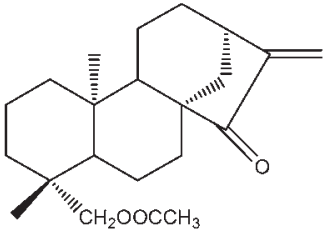

24
Fig. 1 Structure of kaurenoic acid (1) and its derivatives.

Table 1 In vitro cytotoxicitives of 1, 3, 5-14, 17-19, and 24 against selected tumor cell lines as $\mathrm{IC}_{50}(\mu \mathrm{M})$.

\begin{tabular}{|llllllllll|}
\hline Compound & SW620 & SW480 & PANC-1 & BxPC-3 & SGC-7901 & Eca-109 & K562 & HL-60 \\
\hline $\mathbf{1}$ & $>100$ & NT & $>100$ & NT & $>100$ & $>100$ & $>100$ & $>100$ \\
\hline $\mathbf{3}$ & 1.01 & NT & 5.60 & NT & 7.37 & NT & 7.53 & 7.91 \\
\hline $\mathbf{5}$ & 0.98 & NT & 3.36 & 3.28 & 4.51 & 1.85 & 0.53 & 1.68 \\
\hline $\mathbf{6}$ & 0.81 & NT & 0.90 & 1.46 & 2.46 & 1.23 & 0.25 & 1.40 \\
\hline $\mathbf{7}$ & 0.97 & NT & 1.64 & 1.48 & 2.53 & 1.24 & 0.38 & 1.40 \\
\hline $\mathbf{8}$ & 0.99 & 1.16 & 1.97 & NT & 1.95 & 1.26 & 2.47 & 0.42 \\
\hline $\mathbf{9}$ & 0.73 & NT & NT & NT & 1.15 & NT & 1.09 & NT \\
\hline $\mathbf{1 0}$ & 0.58 & NT & NT & NT & 2.65 & NT & 0.93 & NT \\
\hline $\mathbf{1 1}$ & 0.70 & 1.13 & 1.22 & NT & 1.63 & 1.92 & 2.09 & 0.93 \\
\hline $\mathbf{1 2}$ & 1.06 & NT & NT & NT & 1.28 & NT & 0.98 & NT \\
\hline $\mathbf{1 3}$ & 0.89 & NT & NT & NT & 1.59 & NT & 1.21 & NT \\
\hline $\mathbf{1 4}$ & 0.87 & NT & NT & NT & 1.23 & NT & 0.96 & NT \\
\hline $\mathbf{1 7}$ & 0.96 & 1.48 & 1.64 & NT & 3.14 & 2.46 & 1.64 & 1.48 \\
\hline $\mathbf{1 8}$ & 0.73 & 1.29 & 1.45 & NT & 2.59 & 1.93 & 1.29 & 1.09 \\
\hline $\mathbf{1 9}$ & 0.70 & 1.14 & 1.45 & NT & 2.19 & 1.91 & 1.60 & 1.25 \\
\hline $\mathbf{2 4}$ & 1.10 & NT & NT & NT & 2.47 & NT & 1.77 & NT \\
\hline Cisplatin & 13.26 & 15.58 & 10.78 & 5.17 & 8.92 & 2.76 & 4.98 & 1.92 \\
\hline
\end{tabular}

$\alpha$ Cell line: SW620 = colon; SW480 = colon; PANC-1 = pancreatic; BxPC-3 = pancreatic; SCC-7901 = stomach; Eca-109 = esophageal; K562 = leukemia; HL-60 = leukemia; NT: not tested

ence their activities. This was yet again evidenced by the cytotoxicity of compounds 17-19 with piperdinethyl, piperdinepropyl, and piperdinebutyl ester groups at the C-4 position. The activity of compounds $\mathbf{1 1}$ and 24 indicated that inversion of the ester bond had little affect on the activity. The SW620 colon cancer cell was highly susceptible to all tested derivatives, and the standard deviation of their average $\mathrm{IC}_{50}$ from compound $\mathbf{3}$ to compound $\mathbf{2 4}$ was $0.16 \mu \mathrm{M}$.
This semi-synthesis of kaurenoic acid derivatives has revealed several compounds with increased cytotoxic activity. Further studies are required to lower toxicity against normal cells and enhance the effect against cancer cell lines. 


\section{Materials and Methods}

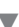

Isolation: The staring material kaurenoic acid [ent-kaur-16-en19-oic acid, (1; 0 Fig. 1)] was isolated from the mangrove-associated plant of $W$. prostrata as previously described [3].

General: IR spectra were measured on a Nicolet FT-IR spectrometer with $\mathrm{KBr}$ pellets. ${ }^{1} \mathrm{H}-\mathrm{NMR}$ and ${ }^{13} \mathrm{C}$-NMR spectra were recorded on a Bruker AVANCE III spectrometer using TMS as the internal standard and $\mathrm{CDCl}_{3}$ as the solvent, reported in Supporting Information. Chemical shifts $(\delta)$ are expressed in ppm with reference to the solvent signals. Column chromatography was performed on silica gel (100-200 mesh and 200-300 mesh; Qingdao Marine Chemical Group Corporation), ESI-MS data were obtained using a Bruker APEX II FT-MS, and EI-MS data was obtained with a ThermoFinnigan DECAX-30000 mass spectrometer. HR-ESI-MS data were obtained with a Bruker APEX II mass spectrometer. Optical rotations were obtained using a JASCO-20 polarimeter. TLC was carried out on silica gel GF254 on glass plates (Qingdao Marine Chemical, Inc.) using various solvent systems. The spots were visualized under UV light or by spraying with $5 \% \mathrm{H}_{2} \mathrm{SO}_{4}$ in EtOH followed by heating. All other reagents were purchased from Aladdin Reagent Company in analytic grade.

Cytotoxic activities against human tumor cell lines including SW620, SW480, PANC-1, BxPC-3, SGC-7901, Eca-109, K562, and HL-60 were evaluated with the MTT assay method [15].

15-Oxo-kaurenoic acid (3) [14]: amorphous solid (36\% yield); m.p. 179.8-180.9 ${ }^{\circ} \mathrm{C}$; IR $(\mathrm{KBr}): v_{\max }=3106,2926,1682,1731 \mathrm{~cm}^{-1}$; ESI-MS: $m / z$ (rel. int.) = $316[\mathrm{M}]^{+}$(95), 317 (26), 301 (27), 148 (60), 91 (53); anal. C 75.94, $\mathrm{H} 8.86$, calcd for $\mathrm{C}_{20} \mathrm{H}_{28} \mathrm{O}_{3}, \mathrm{C} 75.91, \mathrm{H}$ 8.92 .

15-Oxo-kaurenoic acid propanamide (5): white foamy solid (19\% yield); m.p. $156.4-157.6^{\circ} \mathrm{C} ;[\alpha]_{\mathrm{D}}^{24}:-13.5\left(\mathrm{CHCl}_{3}, c\right.$ 0.10); IR ( $\left.\mathrm{KBr}\right)$ : $V_{\text {max }}=3380,2926,2860,1720,1638,1517,1463 \mathrm{~cm}^{-1}$; HRESI-MS: $[\mathrm{M}+\mathrm{Na}]^{+} m / z=380.2568$ (calcd. for $\mathrm{C}_{23} \mathrm{H}_{35} \mathrm{NO}_{2} \mathrm{Na}$ : 380.2566); EI-MS: $m / z$ (rel. int.) = $357\left[\mathrm{M}^{+}\right.$(90), 358 (23), 329 (95), 228 (76).

15-Oxo-kaurenoic acid isopropyl amide (6): white foamy solid (24\% yield); $[\alpha]_{\mathrm{D}}^{24}:-12.9\left(\mathrm{CHCl}_{3}, c \quad 0.10\right)$; IR (KBr): $v_{\max }=3418$, 2958, 2860, 1724, 1632, 1518, $1448 \mathrm{~cm}^{-1}$; HR-ESI-MS: [M + Na $]^{+}$ $m / z=380.2568$ (calcd. for $\mathrm{C}_{23} \mathrm{H}_{35} \mathrm{NO}_{2} \mathrm{Na}$ : 380.2566); ESI-MS: $m / z$ (rel. int.) = $357[\mathrm{M}]^{+}$(100), 358 (26), 329 (97) 228 (48).

15-Oxo-kaurenoic acid butyramide (7): white foamy solid (21\% yield); $[\alpha]_{\mathrm{D}}^{24}:-13.9\left(\mathrm{CHCl}_{3}, c\right.$ 0.10); IR (KBr): $v_{\max }=3375,2915$, 2854, 1726, 1632, 1512, $1452 \mathrm{~cm}-1$; HR-ESI-MS: $[\mathrm{M}+\mathrm{Na}]^{+} \mathrm{m} /$ $z=394.2722$ (calcd. for $\mathrm{C}_{24} \mathrm{H}_{37} \mathrm{NO}_{2} \mathrm{Na}$ : 394.2723); EI-MS: $\mathrm{m} / z$ (rel. int.) = $371[\mathrm{M}]^{+}$(56), 343 (60), 228 (45), 209 (48) 142 (100). 15-Oxo-kaurenoic acid benzoylamide (8): white foamy solid (20\% yield); $[\alpha]_{\mathrm{D}}^{24}:-14.9\left(\mathrm{CHCl}_{3}, c 0.10\right)$; m.p. $210.2-211.6^{\circ} \mathrm{C}$; IR ( $\left.\mathrm{KBr}\right)$ : $v_{\max }=3386,2920,2849,1720,1638,1517,1452,1249 \mathrm{~cm}^{-1}$; HR-ESI-MS: $[\mathrm{M}+\mathrm{Na}]^{+} m / z=428.2567\left(\right.$ calcd. for $\mathrm{C}_{27} \mathrm{H}_{35} \mathrm{NO}_{2} \mathrm{Na}$ : 428.2566); ESI-MS: $m / z$ (rel. int.) $=405[\mathrm{M}]^{+}$(52), 377 (34), 243 (38), 228 (41).

15-Oxo-kaurenoic acid phenylalanine methyl ester amide (9): white amorphous solid (20\% yield); $[\alpha]_{\mathrm{D}}^{24}:-11.7\left(\mathrm{CHCl}_{3}, c 0.10\right)$; IR (KBr): $v_{\max }=3408,2942,2855,1726,1643,1517 \mathrm{~cm}^{-1}$; HR-ESI-MS: $[\mathrm{M}+\mathrm{Na}]^{+} m / z=500.2771$ (calcd. for $\mathrm{C}_{30} \mathrm{H}_{39} \mathrm{NO}_{4} \mathrm{Na}$ : 500.2777); ESI-MS: $m / z$ (rel. int.) $=477[\mathrm{M}]^{+}(100), 423$ (5), 380 (6).

15-Oxo-kaurenoic acid valine methyl ester amide (10): white amorphous solid (22\% yield); $[\alpha]_{\mathrm{D}}^{24}$ : $-15.2\left(\mathrm{CHCl}_{3}\right.$, c 0.10); IR ( $\left.\mathrm{KBr}\right)$ : $V_{\max }=3413,2936,2866,1736,1720,1660,1506,1194 \mathrm{~cm}^{-1}$;
HR-ESI-MS: $[\mathrm{M}+\mathrm{Na}]^{+} m / z=452.2783$ (calcd. for $\mathrm{C}_{26} \mathrm{H}_{39} \mathrm{NO}_{4} \mathrm{Na}$ : 452.2777); ESI-MS: $m / z$ (rel. int.) $=429\left[\mathrm{M}^{+}(100), 398\right.$ (8), 380 (6).

15-Oxo-kaurenoic acid ethyl ester (11): amorphous solid (27\% yield), m.p. $148.5-149.6^{\circ} \mathrm{C} ;[\alpha]_{\mathrm{D}}^{24}$ : $-14.5\left(\mathrm{CHCl}_{3}\right.$, c 0.10$)$; IR: ( $\left.\mathrm{KBr}\right)$ $v_{\max }=3426,2950,2926,1728,1692,1452,1245 \mathrm{~cm}^{-1}$; HRESI-MS: $[\mathrm{M}+\mathrm{Na}]^{+} m / z=367.2253$ (calcd. for $\mathrm{C}_{22} \mathrm{H}_{32} \mathrm{O}_{3} \mathrm{Na}$ : 367.2249); EI-MS: $m / z$ (rel. int.) = $344[\mathrm{M}]^{+}$(100), 329 (11), 271 (48), 91 (37).

15-Oxo-kaurenoic acid propyl ester (12): amorphous solid (23\% yield); $[\alpha]_{\mathrm{D}}^{24}:-6.4\left(\mathrm{CHCl}_{3}, c\right.$ 0.10); IR: $(\mathrm{KBr}) v_{\max }=3426,2955$, 2926, 1727, 1692, 1456, $1245 \mathrm{~cm}^{-1}$; HR-ESI-MS: [M + Na $]^{+} \mathrm{m} /$ $z=382.2400$ (calcd. for $\mathrm{C}_{23} \mathrm{H}_{34} \mathrm{O}_{3} \mathrm{Na}$ : 381.2406); EI-MS: $\mathrm{m} / z$ (rel. int. $)=359[\mathrm{M}+\mathrm{H}]^{+}(100), 343$ (16), 318 (5).

15-Oxo-kaurenoic acid butyl ester (13): amorphous solid (25\% yield); $[\alpha]_{\mathrm{D}}^{24}:-14.1\left(\mathrm{CHCl}_{3}, c 0.10\right) ; \mathrm{IR}(\mathrm{KBr}): v_{\max }=3428,2955$, 2926, 1729, 1692, 1456, $1244 \mathrm{~cm}^{-1}$; HR-ESI-MS: $[\mathrm{M}+\mathrm{Na}]^{+} \mathrm{m} /$ $z=395.2565$ (calcd. for $\mathrm{C}_{24} \mathrm{H}_{36} \mathrm{O}_{3} \mathrm{Na}$ : 395.2562); EI-MS: $\mathrm{m} / z$ (rel. int.) $=373[\mathrm{M}+\mathrm{H}]^{+}(100), 357(6), 318(5)$.

15-Oxo-kaurenoic acid benzyl ester (14): amorphous solid (31\% yield); $[\alpha]_{\mathrm{D}}^{24}:-17.8\left(\mathrm{CHCl}_{3}, c\right.$ 0.10); IR (KBr): $v_{\max }=3418,2942$, 2855, 1721, 1638, 1522, $1241 \mathrm{~cm}^{-1}$; HR-ESI-MS: $[\mathrm{M}+\mathrm{Na}]^{+} \mathrm{m} /$ $z=429.2403$ (calcd. for $\mathrm{C}_{27} \mathrm{H}_{34} \mathrm{O}_{3} \mathrm{Na}$ : 429.2406); EI-MS: $\mathrm{m} / z$ (rel. int.) $=407[\mathrm{M}+\mathrm{H}]^{+}(100), 389$ (13), 338 (33), 315 (50).

15-Oxo-kaurenoic acid piperdinethyl ester (17): yellow oily liquid (17\% yield); $[\alpha]_{\mathrm{D}}^{24}:-11.9\left(\mathrm{CHCl}_{3}\right.$, c 0.10$)$; IR (KBr): $v_{\max }=2931$, 2854, 1721, 1638, 1457, 1221, $1162 \mathrm{~cm}^{-1}$; HR-ESI-MS: [M + Na ${ }^{+}$ $m / z=450.2979$ (calcd. for $\mathrm{C}_{27} \mathrm{H}_{41} \mathrm{NO}_{3} \mathrm{Na}$ : 450.2984); ESI-MS: $m / z$ (rel. int.) $=427[\mathrm{M}]^{+}$(5), 111 (34), 98 (100).

15-Oxo-kaurenoic acid piperdinepropyl ester (18): yellow oily liquid (18\% yield); $[\alpha]_{\mathrm{D}}^{24}$ : $-12.2\left(\mathrm{CHCl}_{3}, c \quad 0.10\right)$; IR (KBr): $v_{\max }=$ 2931, 2849, 1721, 1643, 1457, 1222, $1156 \mathrm{~cm}^{-1}$; HR-ESI-MS: [M $+\mathrm{H}]^{+} m / z=442.3322$ (calcd. for $\mathrm{C}_{28} \mathrm{H}_{44} \mathrm{NO}_{3}$ : 442.3321); ESI-MS: $m / z$ (rel. int.) $=441[\mathrm{M}]^{+}(8), 413$ (4), 149 (15), 98 (100).

15-Oxo-kaurenoic acid piperdinebutyl ester (19): yellow oily liquid (21\% yield); $[\alpha]_{\mathrm{D}}^{24}:-12.7\left(\mathrm{CHCl}_{3}, c \quad 0.10\right)$; $\mathrm{IR}(\mathrm{KBr}): v_{\max }=$ 2926, 2849, 1720, 1671, 1469, 1436, 1227, $1151 \mathrm{~cm}^{-1}$; HRESI-MS: $[\mathrm{M}+\mathrm{Na}]^{+} \mathrm{m} / z=478.3293$ (calcd. for $\mathrm{C}_{29} \mathrm{H}_{45} \mathrm{NO}_{3} \mathrm{Na}$ : 478.3297); EI-MS: $m / z$ (rel. int.) $=455[\mathrm{M}]^{+}(8), 156$ (10), 98 (100). ent-15-Oxo-kaur-16-en-19-acetoxy (24): white crystals (19\% yield); m.p. $156.4-157.6^{\circ} \mathrm{C}$; $[\alpha]_{\mathrm{D}}^{24}$ : $-12.0\left(\mathrm{CHCl}_{3}, c\right.$ 0.10); IR ( $\left.\mathrm{KBr}\right)$ : $v_{\max }=2932,2834,1732,1638,1446,1391,1238,1024 \mathrm{~cm}^{-1}$; HR-ESI-MS: $[\mathrm{M}+\mathrm{Na}]^{+} \mathrm{m} / z=367.2244$ (calcd. for $\mathrm{C}_{22} \mathrm{H}_{32} \mathrm{O}_{3} \mathrm{Na}$ : 367.2249); EI-MS: $m / z$ (rel. int.)=344 [M] $]^{+}$(100), 318 (83), 304 (42).

The MTT assay was performed in 96-well plates. Test cells at the $\log$ phase of their growth cycle $\left(3 \times 10^{4} \mathrm{cell} / \mathrm{mL}\right)$ were added to each well $(100 \mu \mathrm{L} /$ well $)$, then treated in three replicates at various concentrations of the samples $(0.1-100 \mu \mathrm{g} / \mathrm{mL})$, and incubated for $24 \mathrm{~h}$ at $37^{\circ} \mathrm{C}$ in a humidified atmosphere of $5 \% \mathrm{CO}_{2}$. After $48 \mathrm{~h}, 20 \mu \mathrm{L}$ of MTT solution ( $5 \mathrm{mg} / \mathrm{mL}$ ) per well were added to each cultured medium, which were then incubated for a further $4 \mathrm{~h}$. Then, DMSO was added to each well $(150 \mu \mathrm{L} /$ well $)$. After $15 \mathrm{~min}$ at room temperature, the OD of each well was measured on a microplate reader at a wavelength of $570 \mathrm{~nm}$. IC $\mathrm{C}_{50}$ values were obtained by a linear regression analysis of percent absorbance versus log drug concentration.

\section{Supporting information}

The ${ }^{1} \mathrm{H}-\mathrm{NMR},{ }^{13} \mathrm{C}-\mathrm{NMR}$, EI-MS, ESI-MS, and HR-MS data of compounds $3, \mathbf{5}-\mathbf{1 4}, \mathbf{1 7}-\mathbf{1 9}$, and $\mathbf{2 4}$, and the general procedures for the synthesis of them are available as Supporting Information. 


\section{Acknowledgements}

$\nabla$

This work was sponsored by the Natural Sciences Foundation of Fujian Province (No.2010J01179), the Science and Technology Project of Fujian Province (No. 2012Y0035), and the Open Project of National Marine Bureau Key Laboratory of Marine Biogenetic Resources (HY201506).

\section{Conflict of Interest}

$\nabla$

The authors declare no conflict of interest.

\section{References}

1 Ohkoshi E, Kamo S, Makino M, Fujimoto Y. Ent-kaurenoic acids from Mikania hirsutissima (Compositae). Phytochemistry 2004; 65: 885-889

2 Lyu JH, Lee GS, Kim KH, Kim HW, Cho SI, Jeong SI, Kim HJ, Ju YS, Kim HK, Sadikot RT, Christman JW, Oh SR, Lee HK, Ahn KS, Joo M. Ent-kaur-16en-19-oic acid, isolated from the roots of Aralia continentalis, induces activation of Nrf2. J Ethnopharmacol 2011; 137: 1442-1449

3 Vieira HS, Takahashi JA, Boaventura MAD. Constituents from aerial parts of Wedelia paludosa. Fitoterapia 2001; 72: 854-856

4 Jiangsu New Medical College. Dictionary of traditional Chinese drugs. Shanghai: Shanghai Science and Technology Press; 2006: 1559-1560

5 Oliveira BH, Sant'Ana AE, Bastos DZ. Determination of the diterpenoid, kaurenoic acid, in Annona glabra by HPLC. Phytochem Anal 2002; 13 : 368-371

6 Davino SC, Giesbrecht AM, Roque NF. Antimicrobial activity of kaurenoic acid derivatives substituted on carbon-15. Braz J Med Biol Res 1988; 22: 1127-1129

7 Wilkens M, Alarcón C, Urzúa A, Mendoza L. Characterization of the bactericidal activity of the natural diterpene kaurenoic acid. Planta Med 2002; 68: 452-454

8 Costa-Lotufo LV, Cunha GM, Farias PA, Viana GS, Cunha KM, Pessoa C, Moraes MO, Silveira ER, Gramosa NV, Rao VS. The cytotoxic and embryotoxic effects of kaurenoic acid, a diterpene isolated from Copaifera langsdorffii oleo-resin. Toxicon 2002; 40: 1231-1234

9 Alonso R, Gomis H, Taddei A, Sajo C. Cytostatic and cytotoxic activity of synthetic diterpene derivatives obtained from (-)-kaur-9(11),16-dien19-oic acid against human cancer cell lines. Lett Drug Des Discov 2005; 2: 255-259

10 Mizokami SS, Arakawa NS, Ambrosio SR, Zarpelon AC, Casagrande R, Cunha TM, Ferreira SH, Cunha FQ Verri WA jr. Kaurenoic acid from Sphag- neticola trilobata inhibits inflammatory pain: effect on cytokine production and activation of the NO-cyclic GMP-protein kinase G-ATP-sensitive potassium channel signaling pathway. J Nat Prod 2012; 75: 896904

11 Okoye TC, Akah PA, Okoli CO, Ezike AC, Omeje EO, Odoh UE. Antimicrobial effects of a lipophilic fraction and kaurenoic acid isolated from the root bark extracts of Annona senegalensis. Evid Based Complement Alternat Med 2012; 2012: 831327

12 Li J, Zhang DY, Wu XM. Synthesis and biological evaluation of novel exomethylene cyclopentanone tetracyclic diterpenoids as antitumor agents. Bioorg Med Chem Lett 2011; 21: 130-132

13 Zeng YF, Wu JQ Shi LY, Wang K, Zhou B, Tang Y, Zhang DY, Wu YC, Hua $W Y, W u$ XM. Synthesis and evaluation of cytotoxic effects of novel $\alpha-$ methylenelactone tetracyclic diterpenoids. Bioorg Med Chem Lett 2012; 22: 1922-1925

14 Hueso-Falcón I, Girón N, Velasco P, Amaro-Luis JM, Ravelo AG, de las Heras $B$, Hortelano $S$, Estevez-Braun $A$. Synthesis and induction of apoptosis signaling pathway of ent-kaurane derivatives. Bioorg Med Chem 2010; 18: 1724-1735

15 Tang M, Shen D, Hu Y, Gao S, Yu S. Cytotoxic triterpenoid saponins from Symplocos chinensis. J Nat Prod 2004; 67: 1969-1974

received November 11, 2014

revised June 22,2015

accepted July 9, 2015

Bibliography

DOI http://dx.doi.org/10.1055/s-0035-1557861

Planta Med Lett 2015; 2: e48-e51

(C) Georg Thieme Verlag KG Stuttgart · New York ·

ISSN 2199-157X

\section{Correspondence}

\section{Yong-hong Zhang}

Key Laboratory of Natural Drug Pharmacology in Fujian Province

School of Pharmacy

Fujian Medical University

88 jiao Tong Road

Fuzhou 350004

P. R. China

Phone: + 8659122862016

Fax: +8659122862016

zhangyh@mail.fjmu.edu.cn 